\title{
The effects of intensity, frequency, static load, and shore hardness on different parameters in vibration sensitivity measurements
}

\author{
PERTTI ERA，VÄINÖ HÄNNINEN \\ From the Research Unit for Sport and Physical Fitness, and the Department of Health Sciences, University of \\ Jyväskylä, Finland
}

SUMMARY The effects of shore hardness, static load, frequency, and intensity of vibration on the amplitude and dynamic force effects of vibration were studied in conditions corresponding to those occurring in vibrotactile sensitivity measurements. The measurements were performed on three silicon plates of known shore hardness. The results indicated that the effect of differences in elasticity on the vibration amplitudes was evident at lower frequencies up to about $200 \mathrm{~Hz}$ whereas at higher frequencies the amplitudes remained at the same level, regardless of elasticity. On dynamic force the effect of elasticity was observed at all frequencies studied $(50-500 \mathrm{~Hz})$. The static load of the contractor had only minimal effects on amplitudes and dynamic force levels at the loads used $(1-3 N)$.

Goldberg and Lindblom ${ }^{1}$ showed the serious problems connected with the Biothesiometer (Bio Medical Instrument Company), one of the earliest commercial instruments for the assessment of vibrotactile thresholds in people. With this instrument the vibrotactile threshold is measured according to the voltage input to the vibrator and the result is later converted into the amplitude of movement of the contactor, which is believed to be proportional to the squared value of the voltage input. As Goldberg and Lindblom's results ${ }^{1}$ indicated, such methodology is open to error owing to differences in the elastic properties of the tissues in different parts of the body and between different subjects. In addition two similar instruments from the same manufacturer gave different results when the measurements were performed on the same subjects and in corresponding circumstances. In spite of these problems reports based on this methodology have also been published quite recently. ${ }^{2}$ In their article Goldberg and Lindblom ${ }^{1}$ introduced a new instrument by which the actual amplitude of contactor movement at threshold level was measured by an acceleration transducer and the frequency of the

Address for reprint requests: P Era, Research Unit for Sport and Physical Fitness, Rautpohjankatu 10, SF-40700 Jyväskylä, Finland.

Received 23 May 1986.

Accepted 23 June 1986 vibration was $100 \mathrm{~Hz}$. They also gave "normal" values at three stimulation sites (tarsal, tibial, and carpal) at different age levels (10-74 years).

The mechanical response to vibratory stimulation is, in addition to the elastic properties of the underlying tissues, also affected by stimulation frequency. ${ }^{3}$ The mechanical response of the tissues is most pronounced at and near the natural frequency of the tissue and becomes lower at both higher and lower frequencies. ${ }^{3}$ Vibrotactile thresholds also depend on the stimulation frequency, as has been demonstrated in numerous studies. In the glabrous skin of the human hand Verrillo ${ }^{4}$ reported the lowest thresholds at about $250 \mathrm{~Hz}$ and higher values at both lower and higher frequencies.

Stimulation intensity in vibration sensitivity measurements has, in later studies, been indicated most often by the amplitude of the movement of the contactor $^{4}$ whereas in older studies vibration intensity was often indicated by pressure or force effects. ${ }^{5}$ As Lynn established in his review 6 the relation between these parameters has not been extensively studied for the conditions used during sensory testing.

The purpose of the present study was to compare the displacement (amplitude) and dynamic force signals with each other and their relation to the voltage input of the vibrator at different frequencies of vibration. The effect of the elastic properties of the 


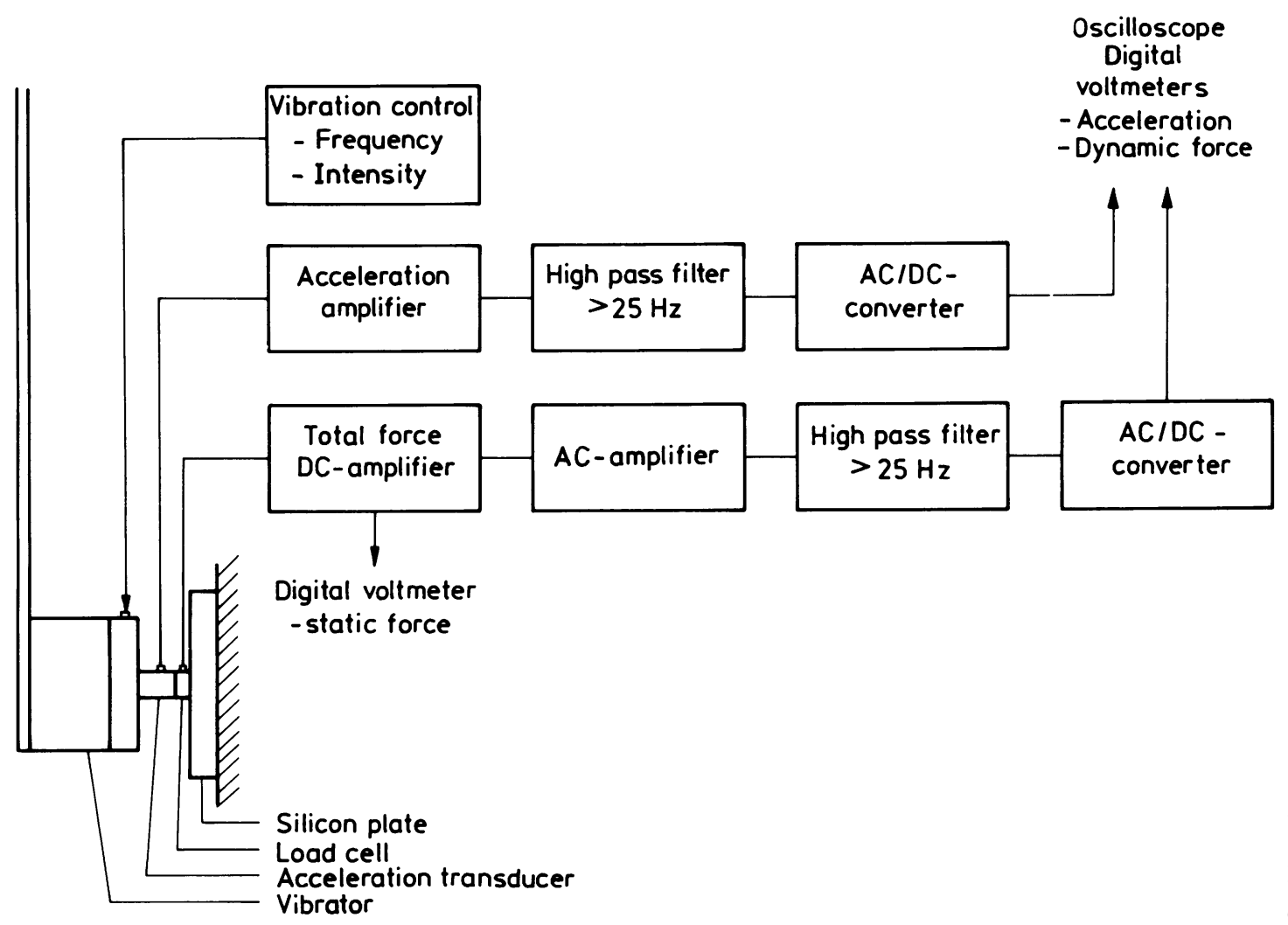

Fig 1 The equipment used in the measurement of acceleration, dynamic force and static load during vibration.

underlying materials on these parameters was studied by making the measurements on three silicon plates of different known shore hardness and by using different static loads on the contactor.

\section{Methods}

The equipment used in the study was composed of a vibrator (Brüel \& Kjær Mini Shaker Type 4810), a piezoelectric acceleration transducer (Brüel \& Kjær Type 4332), a charge amplifier for the acceleration signal (Brüel \& Kjær 2635), a strain gauge force transducer (Miniature Load Cell), DC amplifier for static and dynamic force signals, a power supply constructed in our laboratory, a multi channel storage oscilloscope (Philips PM 3310), standard digital DC/AC meters, and a stand (fig 1).

The silicon plates on which the measurements were performed were cast from materials that differed significantly in their shore hardness. The silicon types were Wacker Silicones RTV-M 533, RTV-M 457 and RTV-ME 426, and their shore A hardness according to the DIN 53505 standard were $25 \pm 3,40 \pm 3$ and $60 \pm 5$ respectively. The thickness of the plates was $10 \mathrm{~mm}$.
During the measurements the plates were fixed on a wall and the contactor was set perpendicularly on the plates by the help of the stand. The area of the circular contactor was $1.33 \mathrm{~cm}^{2}$. The static load of the contactor was controlled by the force transducer and adjusted by a counter-weight.

The effects of the voltage input to the vibrator and of the elasticity of the plates on the dynamic force and acceleration signals was studied at standard frequencies $(50,100,250$ and $500 \mathrm{~Hz})$ and with different static loads $(1,2$ and $3 \mathrm{~N})$ on the plates. The intensity of the vibration was increased step by step from $25 \mathrm{mV}$ to $350 \mathrm{mV}$ and the forms of the dynamic force and acceleration signals were controlled by the oscilloscope. Only measurements where signals followed the sineform were accepted.

The effects of vibration frequency and the elasticity of the plates on the dynamic force and acceleration signals at different static loads were studied by keeping the voltage input to the vibrator at the same level $(100 \mathrm{mV})$ and by increasing the frequency at ascending intervals from $50 \mathrm{~Hz}$ to $500 \mathrm{~Hz}$. Here also the quality of the results was controlled by the oscilloscope.

The amplitude of the movement of the contactor was calculated afterwards from the acceleration values taking into account the frequency of vibration in each set of conditions. 


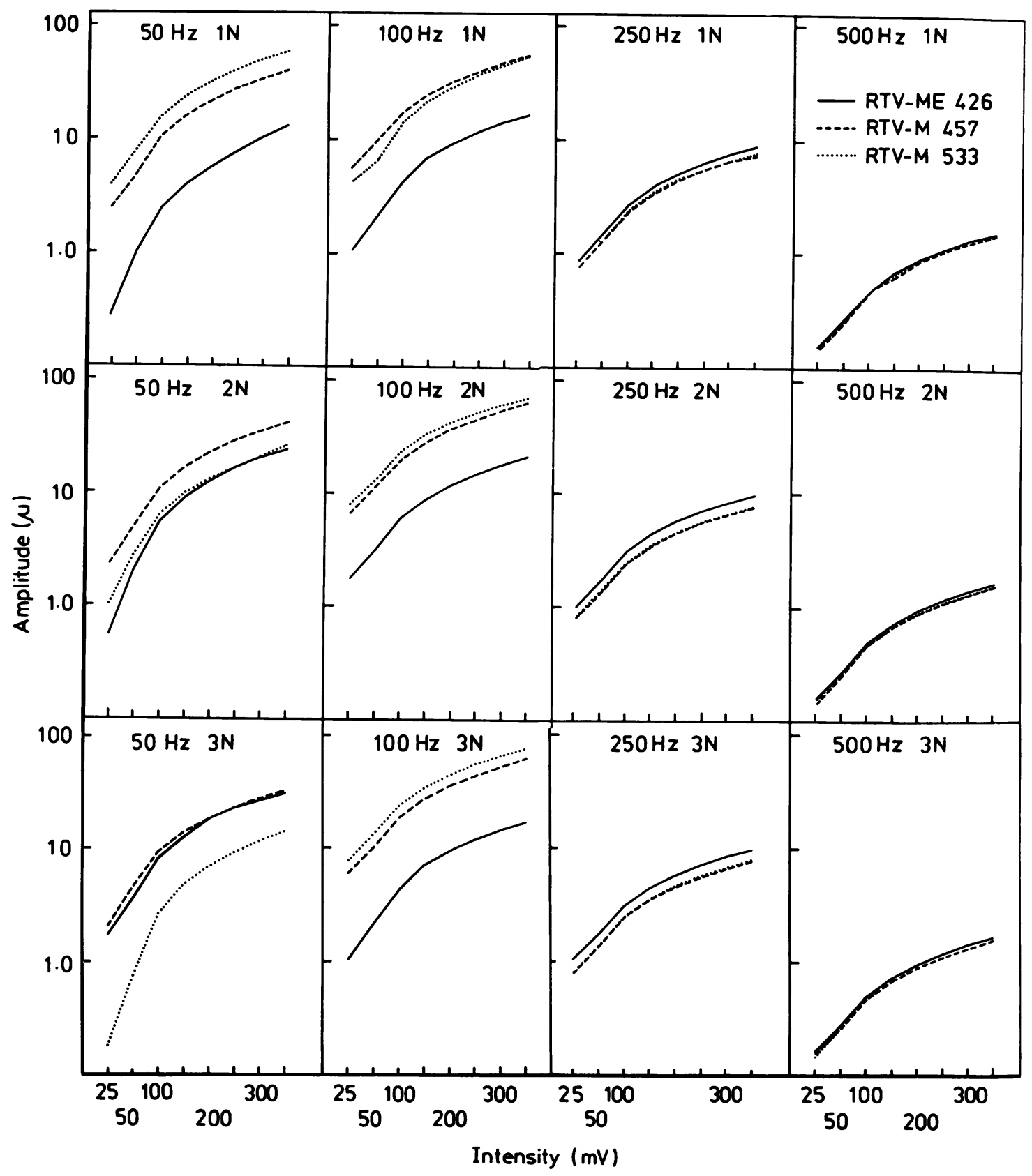

Fig 2 Vibration amplitudes at different frequencies and intensities on the three silicon plates of different shore hardness (RMS-values). Each row corresponds to a constant static load on the contactor (1,2 and $3 \mathrm{~N}$ ). 


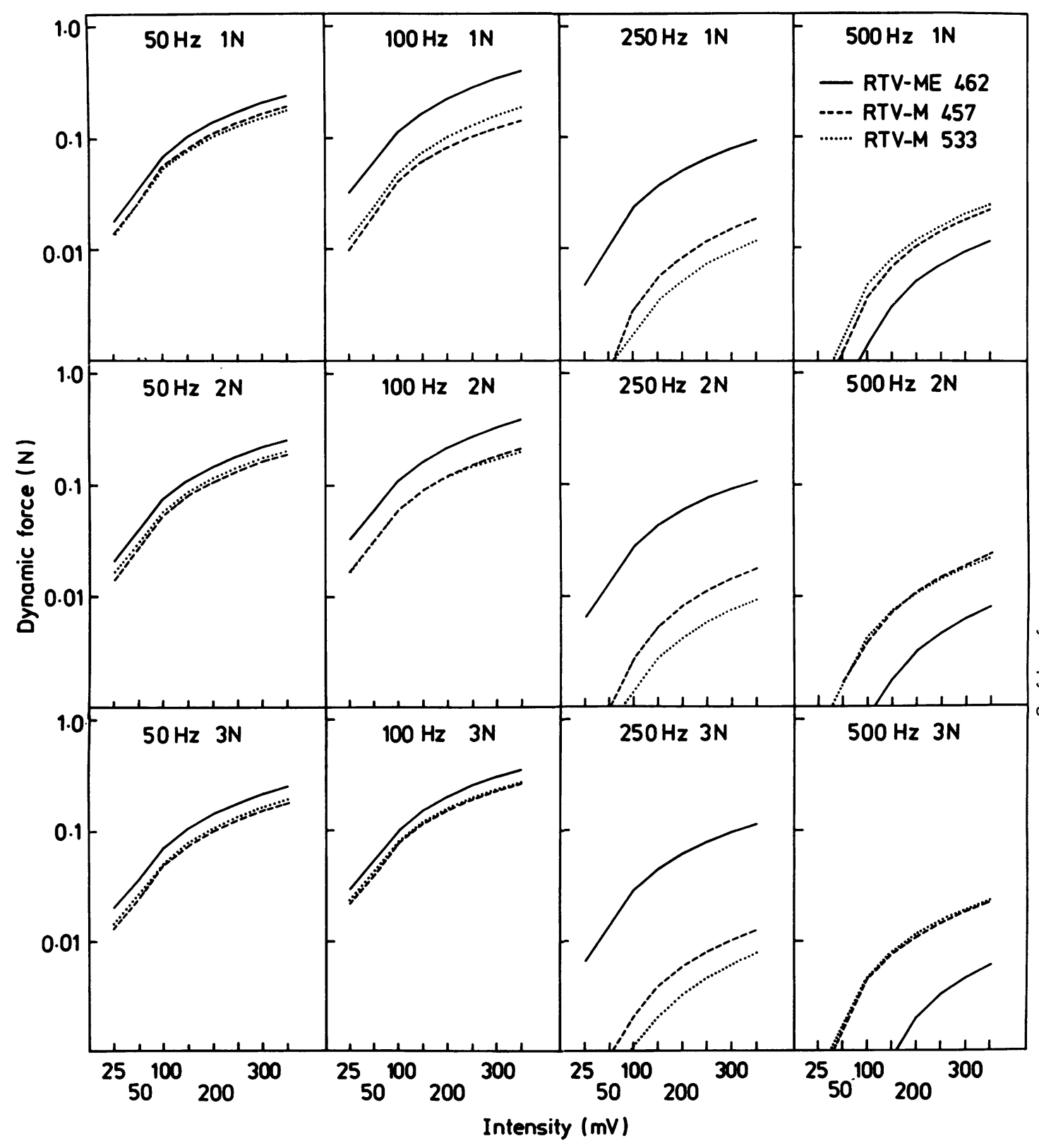

Fig 3 The dynamic force effects of the vibration at different frequencies and intensities on the three silicon plates of different shore hardness (RMS-values). Each row corresponds to a constant static load on the contactor (1,2 and $3 \mathrm{~N}$ ). 
Results

The amplitude of the movement of the contactor was related to the shore hardness of the silicon plates at lower frequencies (fig 2). On the hardest and least elastic plate (plate RTV-ME 426) the amplitude of the vibration was significantly lower than on more elastic materials (plates RTV-ME 533 and RTV-ME 457) both at low and at high intensities of vibration at $50 \mathrm{~Hz}$ and $100 \mathrm{~Hz}$.

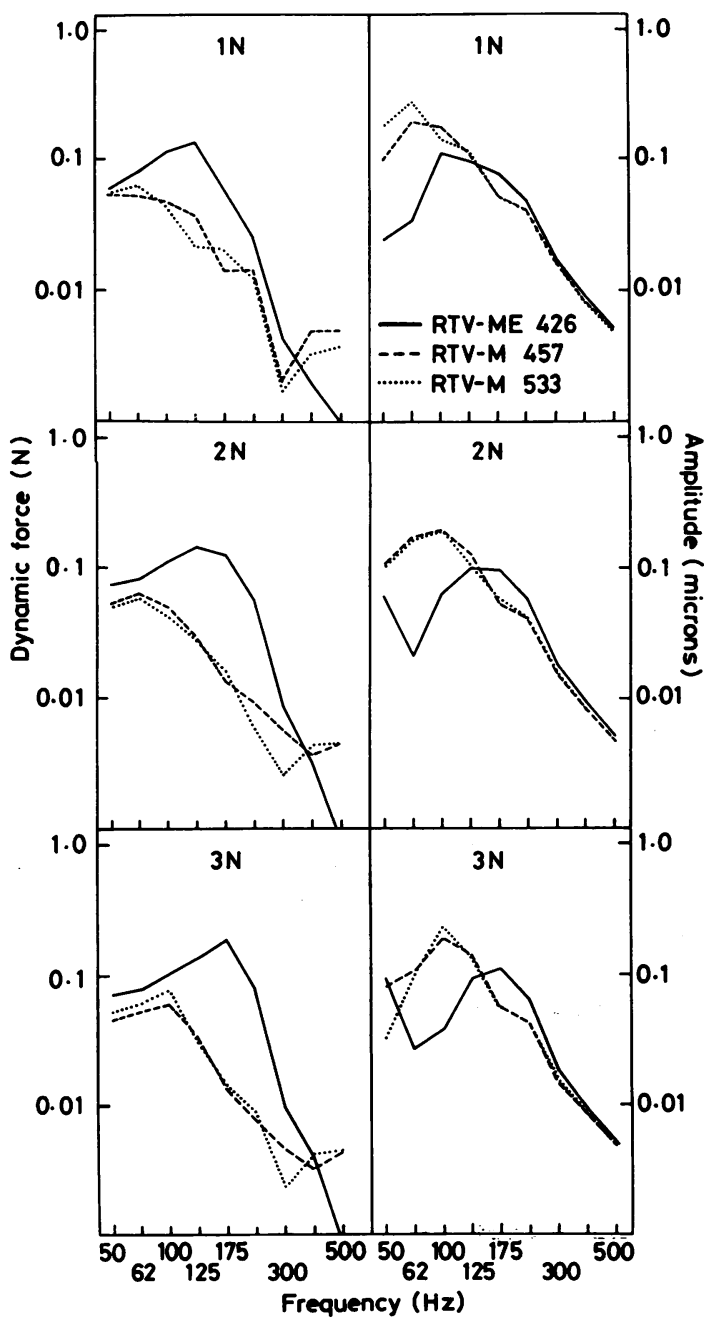

Fig 4 The dynamic force effects (left column) and amplitudes (right column) at different vibration frequencies on the three silicon plates of different shore hardness (RMS-values). Vibration intensity is constant $(100 \mathrm{mV}$ input to the vibrator). Each row corresponds to a constant static load on the contactor $(1,2$ and $3 N)$.
At the higher frequencies of vibration (250 and $500 \mathrm{~Hz}$ ) the differences between the amplitudes on different plates disappeared at the same time as the absolute values of the amplitudes naturally became lower. This evident similarity in the mechanical responses as indicated by the displacement values at the higher frequencies is even more marked when the logarithmic scale in the amplitude values in fig 2 is taken into account. The static load of the contactor did not have any significant effect on the displacement values within the range of static loads used in the measurements $(1-3 \mathrm{~N})$.

The elasticity of the plates also had significant effect on the dynamic force values (fig 3 ).

The dynamic force values were highest on the least elastic material (plate RTV-ME 426) at 50, 100 and $250 \mathrm{~Hz}$. At $500 \mathrm{~Hz}$, where the force signals were, in absolute values, at a very low level on all plates, the hardest material gave the lowest values. Differences in the mechanical responses of the plates as indicated by the dynamic force values could thus be observed at both lower and higher frequencies and at low and higher intensities of vibration. The level of the static load of the contactor did not significantly affect the dynamic force signals.

The effects of the frequency of vibration and of the elasticity of the underlying material on the displacement and dynamic force values was finally studied by keeping the intensity of the vibration constant at $100 \mathrm{mV}$ input to the vibrator and by increasing the frequency at ascending intervals from $50 \mathrm{~Hz}$ to $500 \mathrm{~Hz}$ (fig 4). Here again, the displacement values on plates of different elasticity seemed to reach a common level at about $200 \mathrm{~Hz}$, whereas in the dynamic force signals differences between the plates could be seen at all frequencies. The dynamic force signals reached the highest level on the hardest material (plate RTV-ME 426) at frequencies from 50 to $400 \mathrm{~Hz}$, and were similar on the-two more elastic plates. Static load had a significant effect on the displacement values only at the lowest frequencies $(50-100 \mathrm{~Hz})$ where the amplitudes on different plates were in a different order depending on the level of the static load.

\section{Discussion}

The results of the present study agree with the observations of Goldberg and Lindblom ${ }^{1}$ indicating that in a vibration measurement at a frequency of $100 \mathrm{~Hz}$ the amplitude of a contactor's movement is also affected, in addition to the intensity of the vibration, by the elastic properties of the underlying materials. Our results show that the effect of shore hardness is also obvious at lower and at higher frequencies up to about $200 \mathrm{~Hz}$ where the differences between the plates begin to be minimal. The frequency level at which the 
differences in the amplitudes due to differences in the elastic properties of the underlying materials begin to reach an insignificant level depends on the mechanical impedances of the latter. In measurements made on human tissues the differences in mechanical impedance in different parts of the body may be even bigger than between the silicon plates used in the present study.

The dynamic force signals during vibration at different frequencies and on different plates were different in nature from the displacement values. Differences in the mechanical responses of the plates as indicated by the dynamic force effects also remained evident at the higher vibration frequencies up to $400-500 \mathrm{~Hz}$. At these high frequencies the absolute values of the force signals were very low and reached the separative ability of the load cell. These observations indicate, that if the measurement of vibration sensitivity is performed following the force effects of vibration, the elastic properties of the underlying tissues have an impact on the force effects at a given intensity of vibration and that these differences probably remain up to rather high frequencies.

When the displacement and dynamic force values were compared with each other it was observed that at lower frequencies $(50-100 \mathrm{~Hz})$ these measures gave rather similar results: at both low and high vibration intensities the least elastic plate gave the highest dynamic force values and lowest amplitudes. At higher frequencies, however, the dynamic force signals still indicated differences in the mechanical responses of the plates, whereas the displacement values were at the same level regardless of the elasticity of the plates.

Differences in the displacement and dynamic force signals were small at different static load levels $(1-3 N)$. Only at the lowest frequencies were differences observed in the displacement values. The range of the static loads used was, on the one hand, probably too narrow to cause significant pressure effects on the plates and on the other hand the lowest static load was also high enough to keep the contactor adequately on the plates during measurements at different intensities. In the vibration sensitivity measurements, however, setting the contactor onto the skin is a critical phase which must be precisely controlled, and this may be done by contactor indentation of the skin. ${ }^{4}$

If the displacement value is an adequate stimulus for the receptors, as is commonly thought, ${ }^{1}$ differences due to the elastic properties of different stimulation sites can be taken into account by using instruments which measure the actual displacement values. If the force or pressure effects are the focus of interest, the results of the present study indicate that in a vibration sensitivity measurement using these parameters they too should be directly measured and cannot be reliably determined according to the voltage input to the vibrator.

This study was supported by grants from the Research Council for Sport and Physical Culture in the Ministry of Education, Finland.

\section{References}

1 Goldberg JM, Lindblom U. Standardised method of determining vibratory perception thresholds for $D$ diagnosis and screening in neurological investigation. J Neurol Neurosurg Psychiatry 1979;42:793-803.

2 Bloom S, Till S, Sönksen P, Smith S. Use of ac̄. biothesiometer to measure individual vibration? thresholds and their variation in 519 non-diabetic subjects. Br Med J 1984;288:1793-5.

3 Lunström R. Local vibrations-mechanical impedance of the human hand's glabrous skin. $J$ Biomech 1984;17:137-44.

4 Verrillo RT. Change in vibrotactile thresholds as a function of age. Sensory Processes 1979;3:49-59.

5 Perret E, Regli R. Age and perceptual threshold for vibratory stimuli. Eur Neurol 1970;4:65-76.

6 Lynn B. Cutaneous sensation. In: Goldsmith LA, ed. Biochemistry and Physiology of the Skin. New York: Oxford University Press, 1983:654-84. 